\title{
Explicit enumeration of triangulations with multiple boundaries
}

\author{
Maxim Krikun \\ Institut Élie Cartan, Université Henri Poincaré \\ B.P. 239, 54506, Vandœuvre-lès-Nancy, France \\ krikun@iecn.u-nancy.fr
}

Submitted: Jun 5, 2007; Accepted: Aug 14, 2007; Published: Aug 27, 2007

Mathematics Subject Classification: 05C30

\begin{abstract}
We enumerate rooted triangulations of a sphere with multiple holes by the total number of edges and the length of each boundary component. The proof relies on a combinatorial identity due to W.T. Tutte.
\end{abstract}

\section{Introduction}

\subsection{Definitions}

A planar map is a class of equivalence of embedded graphs $G \hookrightarrow S^{2}$ by the homeomorphisms of $S^{2}$. We note by $V(G), E(G)$ and $F(G)$ the sets of vertices, edges and faces of the the map $G$, respectively.

A map with holes is a pair $(G, H), H \subset F(G)$, such that no two faces $h, h^{\prime} \in H$ share a common vertex, and all vertices at the boundary of $h_{i} \in H$ are distinct (i.e. the boundary of $h_{i}$ is a simple cycle). In the following we refer to the faces $h \in H$ as holes. A map is called a triangulation, if every face of $F(G) \backslash H$ has degree 3. If $H=\emptyset$, such triangulation is called a complete triangulation. In the following we will consider rooted triangulations, that is triangulations with one distinguished directed edge, called the root. In addition to that, we assume that the holes of a triangulation are enumerated by integers $0, \ldots, r$ and that the root is always located at the boundary of the 0 -th hole.

\subsection{Main result}

In this paper we solve explicitly the recursive equations for generating functions planar triangulations with arbitrary number of holes, in terms of the total number of edges and the length of each boundary component. 
The class of triangulations we consider is the most wide possible - the underlying graph may contain multiple edges and loops. Although this class is sometimes thought of as "pathological", it turns out that the presence of loops is a feature which greatly simplifies the calculations involved (e.g. compared to [6]).

Our main result is the following

Theorem 1 Let $C_{r}\left(n, \alpha_{0} ; \alpha_{1}, \ldots, \alpha_{r}\right)$ be the number of rooted triangulations with $(r+1)$ hole, with $\alpha_{j}$ edges on the boundary of the $j$-th hole and $n$ edges in total. Then we have, letting $m=\alpha_{0}+\ldots+\alpha_{r}$,

$$
C_{r}\left(n, \alpha_{0} ; \alpha_{1}, \ldots, \alpha_{r}\right)=\frac{4^{k}(2 m+3 k-2) ! !}{(k+1-r) !(2 m+k) ! !} \alpha_{0} \prod_{j=0}^{r}\left(\begin{array}{c}
2 \alpha_{j} \\
\alpha_{j}
\end{array}\right),
$$

if $n=2 m+3 k$, and

$$
C_{r}\left(n, \alpha_{0} ; \alpha_{1}, \ldots, \alpha_{r}\right)=0
$$

if $n-2 m \neq 0(\bmod 3)$.

\subsection{Related work}

The case $r=0$ corresponds to the problem of enumeration of planar near-triangulations, solved by Tutte in [9] using the method of recursive decomposition. The same method, applied to the problem of enumeration of triangulations on an orientable surface of genus $g$, leads in a natural way to enumeration of triangulations (or maps) with multiple holes. We were unable to obtain any general result in the non-planar case, but for completeness we provide the corresponding recurrent relations in Section 2.3, as well as the generating functions for the triangulations of orientable a torus and double torus $(g=1$ and $g=2)$.

The decomposition method used in our study and the equations involved are not new. The similar ideas were applied by Bender and Canfield ([2]), and later by Arqués and Gioretti ([1]), to the asymptotical enumeration of arbitrary rooted maps on surfaces, and by Gao $([3,4])$ to the asymptotical enumeration of triangulations.

Similar equations appear under the name of loop, or Schwinger-Dyson equations in some models of two-dimensional quantum gravity. Ambjørn et al. studied the asymptotical number of triangulations (and some more general classes of maps) on the sphere and higher genera surfaces with multiple holes (see Chapter 4 in [5]). We have found that the Proposition 1 in section 4 below looks very similar to the formula (4.95) in [5], which expresses the generating function of planar maps with multiple boundary components via the repeated application of the so-called loop insertion operator.

A simplified version of loop insertion operation may be described as follows. Given a complete rooted triangulation, one can cut it along the root edge, and identify the obtained hole with two edges of an additional triangle. This operation provides a bijection between the complete rooted triangulations with $n$ edges, and triangulations with $n+2$ edges and a single hole of length 1 . Thus taking $C_{0}(n+2,1)$, we recover the formula

$$
C_{0}(n+2,1)=\frac{2 \cdot 4^{k-1}(3 k) ! !}{(k+1) !(k+2) ! !}, \quad n=2+3 k,
$$


which gives, by duality, the number of almost trivalent maps with $k$ vertices (sequence A002005 in [8]), computed by Mullin, Nemeth and Schellenberg in [7].

\subsection{Plan of the paper}

This paper is organized as follows. In section 2 we describe the recursive decomposition procedure for triangulations and derive equations on the corresponding generating functions, then solve explicitly these equations for $r=0,1,2,3$. In Section 3 we calculate explicitly the coefficients $C_{r}$ for $r=0,2$. This allows to suggest the main formula of Theorem 1, which is then proved in section 4. The proof closely follows that of [10].

\section{Recurrent relations}

\subsection{Planar triangulations with holes}

Let $C_{k}\left(n, m ; \alpha_{1}, \ldots, \alpha_{k}\right)$ be the number of rooted planar triangulations with $(k+1)$ holes $H=\left(h_{0}, h_{1}, \ldots, h_{k}\right)$, such that there are $m$ edges at the boundary of $h_{0}, \alpha_{j}$ edges on the boundary of $h_{j}, j=1, \ldots, k$ and $n$ edges total.

First, let us remind the recursive decomposition method. Given a rooted planar triangulation $G$ with one hole (that is, a triangulation of a disk), and assuming that there is at least one triangle, one deletes the triangle $t_{0}$ that contains the root. In function of the position of a vertex $v_{0}$, opposite to the root edge in $t_{0}$, there are two possibilities:

(A) if $v_{0}$ is an internal vertex of the triangulation, one obtains a new triangulation with one face less and one more edge on the boundary.

(B) if $v_{0}$ lies on the boundary of $G$, one cuts the resulting map in two parts, with $\left(n_{1}, n_{2}\right)$ edges and the boundaries of length $\left(m_{1}, m_{2}\right)$, such that $n_{1}+n_{2}=n-1$ and $m_{1}+m_{2}=m+1,(n, m)$ being the number of edges and boundary length of the original configuration.

As the final object one obtains a planar map, consisting of a single edge, which we treat as a triangulation with 0 faces, 1 edge and one hole with boundary length 2 .

Now if $G$ is a triangulation with multiple $(k+1$, say) holes, there exists a third possibility for $v_{0}$, namely

(C) if $v_{0}$ is located at the boundary of the hole $h_{j}$, then after erasing the root edge one cuts the resulting map along $v_{0}$, obtaining a map with one hole less, and with boundary of $h_{0}$ having length $m^{\prime}=m+\alpha_{j}+1, \alpha_{j}$ being the length of the boundary of $h_{j}$ in the original triangulation.

Now let $U_{k}\left(x, y, z_{1}, \ldots, z_{k}\right)$ be the multivariate generating function

$$
U_{k}\left(x, y, z_{1}, \ldots, z_{k}\right)=\sum_{N \geq 1} \sum_{m \geq 1} \sum_{\alpha_{j} \geq 1} C\left(n, m ; \alpha_{1}, \ldots, \alpha_{k}\right) x^{n} y^{m} z_{1}^{\alpha_{1}} \cdots z_{k}^{\alpha_{k}}
$$


Translating the above decomposition procedure into the language of generating function, we get the following

Lemma 2.1 The following equations hold

$$
\begin{aligned}
U_{0}(x, y)= & x y^{2}+\frac{x}{y}\left(U_{0}(x, y)-y L_{0}(x)\right)+\frac{x}{y} U_{0}^{2}(x, y) \\
U_{k}(x, y ; \mathbf{z})= & \frac{x}{y}\left(U_{k}(x, y ; \mathbf{z})-y L_{k}(x ; \mathbf{z})\right) \\
& +\frac{x}{y} \sum_{\omega \subset I_{k}} U_{|\omega|}\left(x, y ; \mathbf{z}_{\omega}\right) U_{k-|\omega|}\left(x, y ; \mathbf{z}_{I_{k} \backslash \omega}\right) \\
& +\sum_{j=1}^{k}\left[\frac{x}{y-z_{j}}\left(\frac{z_{j}}{y} U_{k-1}\left(x, y ; \hat{\mathbf{z}}_{j}\right)-\frac{y}{z_{j}} U_{k-1}\left(x, z_{j} ; \hat{\mathbf{z}}_{j}\right)\right)+x L_{k-1}\left(x ; \hat{\mathbf{z}}_{j}\right)\right]
\end{aligned}
$$

where

$$
L_{k}(x ; \mathbf{z})=[y] U_{k}(x, y ; \mathbf{z}),
$$

$I_{k}=\{1,2, \ldots, k\}$ is the index set, the sum is over all subsets $\omega$ of $I_{k}$ (including empty set and $I_{k}$ itself), $\mathbf{z}$ stands for $z_{1}, \ldots, z_{k}, \mathbf{z}_{\omega}$ is the list of variables $z_{j}$ with $j \in \omega$, and $\hat{\mathbf{z}}_{j}$ stands for $z_{1}, \ldots, z_{k}$ without $z_{j}$.

Proof. The equation (2) is a classical relation for the generating function of neartriangulations: the term $x y^{2}$ accounts for the special single-edged map, the term, linear in $U_{0}$, corresponds to the case (A) above, and the quadratic term to the case (B).

In (3), the first term on the right-hand side is derived exactly the same way as in (2); the summation over $\omega$ corresponds to the possible ways to distribute the $k$ enumerated holes between the two parts of a triangulation in case (B).

To see how the summation over $j$ in (3) arises, consider first the case $k=1$, i.e. a triangulation with two holes. When the rule (C) above applies, removing of the root edge merges the two holes, of lengths $\alpha_{0}$ and $\alpha_{1}$, into a single hole of length $\left(\alpha_{0}+\alpha_{1}+1\right)$. This gives the following contribution to $U_{1}(x, y, z)$ :

$$
\begin{aligned}
& \sum_{n \geq 0} \sum_{\alpha_{0} \geq 1, \alpha_{1} \geq 1} C_{0}\left(n-1, \alpha_{0}+\alpha_{1}+1\right) x^{n} y^{\alpha_{0}} z^{\alpha_{1}} \\
& =x \sum_{n \geq 0} \sum_{m \geq 3} C_{0}(n-1, m) x^{n-1}\left(y z^{m-2}+y^{2} z^{m-3} \ldots+y^{m-2} z\right) \\
& =x \sum_{n \geq 0} \sum_{m \geq 3} C_{0}(n-1, m) x^{n-1} \frac{z y^{m-1}-y z^{m-1}}{y-z} \\
& =\frac{x}{y-z}\left[\frac{z}{y}\left(U_{0}(x, y)-U_{0}(x, 0)-y[t] U_{0}(x, t)-y^{2}\left[t^{2}\right] U_{0}(x, y)\right)\right. \\
& \left.\quad-\frac{y}{z}\left(U_{0}(x, z)-U_{0}(x, 0)-z[t] U_{0}(x, t)-z^{2}\left[t^{2}\right] U_{0}(x, y)\right)\right] \\
& =\frac{x}{y-z}\left(\frac{z}{y} U_{0}(x, y)-\frac{y}{z} U_{0}(x, z)\right)+x[t] U_{0}(x, t) .
\end{aligned}
$$

A general case $k \geq 1$ follows similarly, since when merging the hole $h_{j}$ with the hole $h_{0}$, all other holes remain intact. 


\subsection{Solution of recurrent equations}

The equations (2), (3) may be solved exactly. First, (2) is solved using the quadratic method, giving

$$
U_{0}(x, y)=\frac{h-y}{2 h} \sqrt{1-4 h^{2} y}-\frac{x-y}{2 x},
$$

where $h=h(x)$ is a positive power series in $x$, satisfying the relation

$$
8 h^{3} x^{2}-h^{2}+x^{2}=0
$$

namely

$$
h(x)=\sum_{k=0}^{\infty} \frac{4^{k}(3 k-1) ! !}{k !(k+1) ! !} x^{3 k+1}=x \sum_{k=0}^{\infty} \frac{2^{k}(3 k-1) ! !}{k !(k+1) ! !}\left(2 x^{3}\right)^{k}
$$

(cf. sequence A078531 in [8]).

Next, one may solve $(3)$ with respect to $L_{k}(x ; \mathbf{z})$ and group the terms containing $U_{k}(x, y ; \mathbf{z})$, obtaining

$$
x L_{k}(x, t ; \mathbf{z})=\frac{1}{y}\left(x-y+2 x U_{0}(x, y)\right) U_{k}(x, y ; \mathbf{z})+W_{k}(x, y ; \mathbf{z}),
$$

where $W_{k}(x, y ; \mathbf{z})$ is the sum of terms in $(3)$, not containing $U_{k}$,

$$
\begin{aligned}
W_{k}(x, y ; \mathbf{z}) & =\frac{x}{y} \sum_{\substack{\omega \subset I_{k} \\
1<|\omega|<k}} U_{|\omega|}\left(x, y ; \mathbf{z}_{\omega}\right) U_{k-|\omega|}\left(x, y ; \mathbf{z}_{I_{k} \backslash \omega}\right) \\
& +\sum_{j=1}^{k}\left[\frac{x}{y-z_{j}}\left(\frac{z_{j}}{y} U_{k-1}\left(x, y ; \hat{\mathbf{z}}_{j}\right)-\frac{y}{z_{j}} U_{k-1}\left(x, z_{j} ; \hat{\mathbf{z}}_{j}\right)\right)+x L_{k-1}\left(x ; \hat{\mathbf{z}}_{j}\right)\right] .
\end{aligned}
$$

Note that the left hand side of (7) does not depend on $y$, and the factor $(x-y+$ $\left.2 x U_{0}(x, y)\right)=(x / h)(h-y) \sqrt{1-4 h^{2} y}$ vanishes at $y=h$, thus

$$
U_{k}(x, y ; \mathbf{z})=h y \frac{W_{k}(x, h ; \mathbf{z})-W_{k}(x, y ; \mathbf{z})}{x(h-y) \sqrt{1-4 h^{2} y}} .
$$

In particular, we have

$$
\begin{aligned}
U_{1}(x, y ; z) & =\frac{1}{2} \frac{z\left(1-\sqrt{1-4 h^{2} y}\right)-y\left(1-\sqrt{1-4 h^{2} z}\right)}{(y-z) \sqrt{1-4 h^{2} y}}, \\
U_{2}\left(x, y ; z_{1}, z_{2}\right) & =\frac{8 h^{5} y\left(1-\sqrt{1-4 h^{2} z_{1}}\right)\left(1-\sqrt{1-4 h^{2} z_{2}}\right)}{\left(1-4 h^{3}\right)\left(1-4 h^{2} y\right)^{3 / 2} \sqrt{1-4 h^{2} z_{1}} \sqrt{1-4 h^{2} z_{2}}}
\end{aligned}
$$

It is somewhat more convenient to consider the "symmetrized" functions

$$
U_{k}^{s y m}\left(x, y ; z_{1}, \ldots, z_{k}\right)=z_{1} \cdots z_{k} \frac{\partial^{k}}{\partial z_{1} \cdots \partial z_{k}} U_{k}\left(x, y ; z_{1}, \ldots, z_{k}\right)
$$


which correspond to adding an additional root on each of the $k$ holes $h_{1}, \ldots, h_{k}$. The functions $U_{k}^{s y m}$ are then symmetric in $\left(y, z_{1}, \ldots, z_{k}\right)$ :

$$
\begin{gathered}
U_{1}^{\text {sym }}(x, y ; z)=\frac{4 h^{4} y z}{\left(\sqrt{1-4 h^{2} y}+\sqrt{1-4 h^{2} z}\right)^{2} \sqrt{1-4 h^{2} y} \sqrt{1-4 h^{2} z}}, \\
U_{2}^{\text {sym }}\left(x, y ; z_{1}, z_{2}\right)=\frac{32 h^{9} y z_{1} z_{2}}{\left(1-4 h^{3}\right)\left(1-4 h^{2} y\right)^{3 / 2}\left(1-4 h^{2} z_{1}\right)^{3 / 2}\left(1-4 h^{2} z_{2}\right)^{3 / 2}} \\
U_{3}^{\text {sym }}\left(x, y ; z_{1}, z_{2}, z_{3}\right)=\frac{3072 h^{14} y z_{1} z_{2} z_{3} \times P_{3}\left(h, y, z_{1}, z_{2}, z_{3}\right)}{\left(1-4 h^{3}\right)^{3}\left(1-4 h^{2} y\right)^{5 / 2} \prod_{j=1}^{3}\left(1-4 h^{2} z_{j}\right)^{5 / 2}},
\end{gathered}
$$

where

$$
\begin{aligned}
P_{3}\left(h, y, z_{1}, z_{2}, z_{3}\right)= & 1-3 \sigma_{(1)}\left(h^{3}, h^{2} y, h^{2} z_{1}, h^{2} z_{2}, h^{2} z_{3}\right) \\
& +8 \sigma_{(1,1)}\left(h^{3}, h^{2} y, h^{2} z_{1}, h^{2} z_{2}, h^{2} z_{3}\right) \\
& -16 \sigma_{(1,1,1)}\left(h^{3}, h^{2} y, h^{2} z_{1}, h^{2} z_{2}, h^{2} z_{3}\right) \\
& +256 h^{11} y z_{1} z_{2} z_{3} .
\end{aligned}
$$

and $\sigma_{(1)}, \sigma_{(1,1)}, \sigma_{(1,1,1)}$ are Schur polynomials.

\subsection{Triangulations of higher genera}

The decomposition procedure extends naturally to the triangulations of genus $g$ with the following essential changes (here, as above, $v_{0}$ denotes the vertex opposite to the rooted edge in the triangle which is removed)

(C') If the vertex $v_{0}$ lies on the boundary of $h_{0}$, the map is separated into two parts, and both the holes and the genus should be distributed between these parts;

(D) It is possible that $v_{0}$ lies on the boundary of $h_{0}$ in such a way that after deleting the triangle $t_{0}$ and cutting the map along $v_{0}$ the map stays connected (imagine the hole $h_{0}$ wrapping around the torus). In such case the resulting map will have genus $g-1$ and one more hole.

Let $T_{g, k}\left(x, y ; z_{1}, \ldots, z_{k}\right)$ be the generating function of triangulations of genus $g$ with $(k+1)$ hole (obviously, $T_{0, k}=U_{k}$ ). The decomposition procedure leads to the recursive relations, similar to the main equation in [2]. 
Lemma 2.2 The following relations hold:

$$
\begin{aligned}
T_{g, k}(x, y ; \mathbf{z})= & \frac{x}{y}\left(T_{g, k}(x, y ; \mathbf{z})-y[t] T_{g, k}(x, t ; \mathbf{z})\right) \\
& +\frac{x}{y} \sum_{i=1}^{g} \sum_{\omega \subset I_{k}} T_{i,|\omega|}\left(x, y ; \mathbf{z}_{\omega}\right) T_{g-i, k-|\omega|}\left(x, y ; \mathbf{z}_{I_{k} \backslash \omega}\right) \\
& +\sum_{j=1}^{k}\left[\frac{x}{y-z_{j}}\left(\frac{z_{j}}{y} T_{g, k-1}\left(x, y ; \hat{\mathbf{z}}_{j}\right)-\frac{y}{z_{j}} T_{g, k-1}\left(x, z_{j} ; \hat{\mathbf{z}}_{j}\right)\right)\right. \\
& \left.\quad+x[t] T_{g, k-1}\left(x, t ; \hat{\mathbf{z}}_{j}\right)\right] \\
& +\left.x \frac{\partial}{\partial t} T_{g-1, k+1}\left(x, y ; z_{1}, \ldots, z_{k}, t\right)\right|_{t=y}
\end{aligned}
$$

Proof. When the case (D) applies, after removing the root edge we get a triangulation with an additional hole, and with a distinguished vertex on the boundary of this hole (the image of $v_{0}$ ). This gives the last term in (15), and the rest is similar to (3).

The equation (15) may be solved analogously to (3). In particular, we find generating function for triangulations of genus 1 and 2 with one hole

$$
\begin{aligned}
& T_{1,0}(x, y)=\frac{\left(1-16 h^{5} y\right) h^{5} y}{\left(1-4 h^{3}\right)^{2}\left(1-4 h^{2} y\right)^{5 / 2}} \\
& T_{2,0}(x, y)=\frac{P_{2,0}(h, y)}{\left(1-4 h^{3}\right)^{7}\left(1-4 h^{2} y\right)^{11 / 2}}
\end{aligned}
$$

where

$$
\begin{aligned}
P_{2,0}(h, y)= & 3 h^{11} y\left(35+184 h^{3}+48 h^{6}\right)\left(1024 h^{11} y^{4}+1024 h^{12} y^{3}-1280 y^{3} h^{9}+1\right) \\
& +128 h^{18} y^{3}\left(545+1488 h^{3}-3216 h^{6}+2560 h^{9}\right) \\
& +64 h^{16} y^{2}\left(-307-480 h^{6}+256 h^{9}+324 h^{3}\right)
\end{aligned}
$$

\section{Extracting exact coefficients}

\subsection{Lagrange inversion}

Letting $h=x \sqrt{1+\zeta}$ and $t=x^{3}$ in (5) we get

$$
\zeta=8 t(1+\zeta)^{3 / 2}
$$

so the Lagrange's inversion theorem applies, and we have, assuming $n=m+3 k$,

$$
\begin{aligned}
{\left[x^{n}\right] h^{m} } & =\left[x^{n-m}\right](h / x)^{m}=\left[t^{k}\right](1+\zeta)^{m / 2} \\
& =\frac{1}{k}\left[\lambda^{k-1}\right]\left\{\frac{m}{2}(1+\lambda)^{m / 2-1}(1+\lambda)^{3 k / 2}\right\} \\
& =\frac{m}{k !} 4^{k} \frac{(m+3 k-2) ! !}{(m+k) ! !} .
\end{aligned}
$$


In particular this gives the formula (6) for $h(x)$.

For $U_{0}$ we have the following series expansion in $y$

$$
U_{0}(x, y)=\frac{h-x+2 h^{3} x}{2 h x} y+\sum_{m=0}^{\infty} \frac{1}{m+1}\left(\begin{array}{c}
2 m \\
m
\end{array}\right)\left(1-\frac{4 m+2}{m+2} h^{3}\right) h^{2 m+1} y^{m+2} .
$$

Letting $n=2 m+3 k, k \geq-1$ and using (19) we obtain

$$
\left[x^{n} y^{m}\right] U_{0}(x, y)=m\left(\begin{array}{c}
2 m \\
m
\end{array}\right) \frac{4^{k}(2 m+3 k-2) ! !}{(k+1) !(2 m+k) ! !}
$$

and $\left[x^{n} y^{m}\right] U_{0}(x, y)=0$ if $n+m \neq 0(\bmod 3)$.

Now note that $U_{2}^{(s y m)}$ has the product form, so the expansion is particularly easy to calculate. First we'll need the coefficients

$$
\begin{aligned}
{\left[x^{n}\right]\left\{\frac{32 h^{9}}{1-4 h^{3}}\left(4 h^{2}\right)^{m-3}\right\} } & =\frac{1}{2} \sum_{j=0}^{\infty} 4^{m+j}\left[x^{n}\right] h^{2 m+3 j+3} \\
& =\frac{1}{8} 2^{2 m+2 k}(2 m+3 k-2) ! ! \sum_{j=1}^{k} \frac{2 m+3 j}{(k-j) !(2 m+2 j+k) ! !} \\
& =\frac{1}{8} 2^{2 m+2 k} \frac{(2 m+3 k-2) ! !}{(k-1) !(2 m+k) ! !} .
\end{aligned}
$$

where $n=2 m+3 k$. Then we obtain

$$
\begin{aligned}
{\left[x^{n} y^{\alpha_{0}} z_{1}^{\alpha_{1}} z_{2}^{\alpha_{2}}\right] U_{2}^{(s y m)}\left(x, y, z_{1}, z_{2}\right) } \\
=\prod_{i=0}^{2} \frac{\left(2 \alpha_{i}-1\right) ! !}{2^{\alpha_{i}-1}\left(\alpha_{i}-1\right) !} \cdot\left[t^{n}\right]\left\{\frac{32 h^{9}}{1-4 h^{3}}\left(4 h^{2}\right)^{m-3}\right\} \\
=2^{m+2 k} \frac{(2 m+3 k-2) ! !}{(k-1) !(2 m+k) ! !} \frac{\left(2 \alpha_{0}-1\right) ! !\left(2 \alpha_{1}-1\right) ! !\left(2 \alpha_{2}-1\right) ! !}{\left(\alpha_{0}-1\right) !\left(\alpha_{1}-1\right) !\left(\alpha_{2}-1\right) !} \\
=\alpha_{0} \alpha_{1} \alpha_{2}\left(\begin{array}{c}
2 \alpha_{0} \\
\alpha_{0}
\end{array}\right)\left(\begin{array}{c}
2 \alpha_{1} \\
\alpha_{1}
\end{array}\right)\left(\begin{array}{c}
2 \alpha_{2} \\
\alpha_{2}
\end{array}\right) \cdot \frac{2^{2 k}(2 m+3 k-2) ! !}{(k-1) !(2 m+k) ! !}
\end{aligned}
$$

where $m=\alpha_{0}+\alpha_{1}+\alpha_{2}, n=2 m+3 k$; the coefficient is $C_{2}(n, \ldots)$ is null if $n-2 m \neq$ $0(\bmod 3)$.

The formulae (20), (21) allow to conjecture the following general formula

$$
\left[x^{n} z_{0}^{\alpha_{0}} z_{1}^{\alpha_{1}} \ldots z_{k}^{\alpha_{2}}\right] U_{k}^{(s y m)}\left(x, z_{0} ; \mathbf{z}\right)=\frac{4^{k}(2 m+3 k-2) ! !}{(k+1-r) !(2 m+k) ! !} \prod_{j=0}^{r} \alpha_{j}\left(\begin{array}{c}
2 \alpha_{j} \\
\alpha_{j}
\end{array}\right)
$$

where $m=\alpha_{0}+\ldots+\alpha_{r}$ and $n=2 m+3 k$.

Clearly, this formula is equivalent to (1), and it further agrees with the above expressions for $U_{1}^{(s y m)}$ and $U_{3}^{(s y m)}$ (as can be seen by calculating explicitly few first terms in the power series expansions of these functions). 


\subsection{The combinatorial identity}

The above expression (22) resembles a formula obtained by Tutte in [10], for the number of slicings with $k$ external faces of degrees $2 n_{1}, \ldots, 2 n_{k}$

$$
\gamma\left(n_{1}, n_{2}, \ldots, n_{k}\right)=\frac{(n-1) !}{(n-k+2) !} \prod_{i=1}^{k} \frac{\left(2 n_{i}\right) !}{n_{i} !\left(n_{i}-1\right) !}
$$

The proof of (23) relies on the following combinatorial identity:

$$
\begin{aligned}
\sum_{\omega \subset I} \mathbf{D}^{|\omega|-k}\left\{\lambda \cdot f_{\omega}\right\} \cdot \mathbf{D}^{|\bar{\omega}|-l}\left\{\mu \cdot f_{\bar{\omega}}\right\} & \\
= & \sum_{\substack{\omega \subset I \\
|\omega|<k}} \sum_{i=0}^{k-1-|\omega|}(-1)^{i}\left(\begin{array}{c}
|\bar{\omega}|-l \\
i
\end{array}\right) \mathbf{D}^{|\bar{\omega}|-l-i}\left\{\mathbf{D}^{-k+|\omega|+i}\left\{\lambda \cdot f_{\omega}\right\} \cdot \mu f_{\bar{\omega}}\right\} \\
& +\sum_{\substack{\omega \subset I \\
|\bar{\omega}|<l}} \sum_{i=0}^{l-1-|\bar{\omega}|}(-1)^{i}\left(\begin{array}{c}
|\omega|-l \\
i
\end{array}\right) \mathbf{D}^{|\omega|-l-i}\left\{\lambda f_{\bar{\omega}} \cdot \mathbf{D}^{-l+|\bar{\omega}|+i}\left\{\mu f_{\bar{\omega}}\right\}\right\} .
\end{aligned}
$$

where $I$ is the set $\{1, \ldots, r\} ; \lambda, \mu, f_{1}, \ldots f_{r}$ are arbitrary (sufficiently often differentiable) functions of a single parameter, say $x, f_{\omega}$ denotes the product $f_{\omega}=\prod\left\{f_{i} \mid i \in \omega\right\}$, and $\mathbf{D}$ stands for the differentiation in $x$. Whenever $\mathbf{D}$ appears with negative index (which can only be in the left-hand side of (24)), it is to be treated as an operation of repeated integration, and it is assumed that the constants of integration are fixed in some way for every $X$ that appears as the argument to $\mathbf{D}^{-1}$, so that $\mathbf{D}^{-1}(X)$ is uniquely defined.

\section{Proof of Theorem 1}

The proof is organized as follows: first we interpret the formula (22) in terms of generating

functions $U_{k}^{(s y m)}$. Then we use the equation (3) and the combinatorial identity (24) to show by induction that all of the generating function have the required form.

Note that in (22)

$$
\frac{4^{k}(2 m+3 k-2) ! !}{(k+1-r) !(2 m+k) ! !}=\frac{k !}{(k+1-r) !} \frac{1}{2 m}\left[x^{2 m+3 k}\right] h^{2 m},
$$

thus we have (with $n=2 m+3 k$ )

$$
\begin{aligned}
C_{r}^{(s y m)}\left(n, \alpha_{0} ; \alpha_{1}, \ldots, \alpha_{r}\right) & =\frac{4^{k}(2 m+3 k-2) ! !}{(k+1-r) !(2 m+k) ! !} \prod_{j=0}^{r} \alpha_{j}\left(\begin{array}{c}
2 \alpha_{j} \\
\alpha_{j}
\end{array}\right) \\
& =\frac{k !}{(k+1-r) !}\left[x^{n}\right] \frac{h^{2 m}}{2 m} \prod_{j=0}^{r} \alpha_{j}\left(\begin{array}{c}
2 \alpha_{j} \\
\alpha_{j}
\end{array}\right) \\
& =\frac{k !}{(k+1-r) !}\left[x^{n} z_{0}^{\alpha_{0}} \cdots z_{r}^{\alpha_{r}}\right] \int_{0}^{h^{2}} \prod_{j=0}^{r} \frac{2 s z_{j}}{\left(1-4 s z_{j}\right)^{3 / 2}} \frac{d s}{2 s}
\end{aligned}
$$


since $m=\alpha_{0}+\ldots+\alpha_{r}$, and

$$
\sum_{\alpha=0}^{\infty} \alpha\left(\begin{array}{c}
2 \alpha \\
\alpha
\end{array}\right) z^{\alpha}=\frac{2 z}{(1-4 z)^{3 / 2}}
$$

On the other hand, from (19) we have

$$
\left[x^{2 m+3 k}\right] h^{2 m}=\left[t^{k}\right](1+\zeta(t))^{m}
$$

(where $\zeta(t)$ is defined by (18)), thus we may continue (25) with

$$
\begin{aligned}
\ldots & =\frac{k !}{(k+1-r) !}\left[t^{k}\right]\left\{\left[z_{0}^{\alpha_{0}} \cdots z_{r}^{\alpha_{r}}\right] \int_{0}^{1+\zeta(t)} \prod_{j=0}^{r} \frac{2 s z_{j}}{\left(1-4 s z_{j}\right)^{3 / 2}} \frac{d s}{2 s}\right\} \\
& =\left[t^{k+1-r}\right]\left(\frac{\partial}{\partial t}\right)^{r-1}\left\{\left[z_{0}^{\alpha_{0}} \cdots z_{r}^{\alpha_{r}}\right] \int_{0}^{1+\zeta(t)} \prod_{j=0}^{r} \frac{2 s z_{j}}{\left(1-4 s z_{j}\right)^{3 / 2}} \frac{d s}{2 s}\right\} .
\end{aligned}
$$

Finally, (22) is equivalent to (assuming $r \geq 1$ )

$$
\begin{aligned}
U_{r}^{(\text {sym })}\left(x ; z_{0}, \ldots, z_{r}\right) & =u_{r}^{(\text {sym })}\left(x^{3} ; x^{2} z_{0}, \ldots, x^{2} z_{r}\right) \\
u_{r}^{(s y m)}\left(t ; z_{0}, \ldots, z_{r}\right) & =t^{r-1}\left(\frac{\partial}{\partial t}\right)^{r-1} \int_{0}^{1+\zeta(t)} \prod_{j=0}^{r} \frac{2 s z_{j}}{\left(1-4 s z_{j}\right)^{3 / 2}} \frac{d s}{2 s} .
\end{aligned}
$$

In the non-symmetric case, a similar calculation gives

$$
\begin{aligned}
& U_{r}\left(x, y ; z_{1}, \ldots, z_{r}\right)=u_{r}\left(x^{3}, x^{2} y ; x^{2} z_{1}, \ldots, x^{2} z_{r}\right) \\
& u_{r}\left(t, y ; z_{1}, \ldots, z_{r}\right)=t^{r-1}\left(\frac{\partial}{\partial t}\right)^{r-1} \int_{0}^{1+\zeta(t)} \frac{y}{(1-4 s y)^{3 / 2}} \prod_{j=1}^{r}\left(\frac{1}{\sqrt{1-4 s z_{j}}}-1\right) d s .
\end{aligned}
$$

Now if we put $\hat{u}_{k}=t^{1-r} u_{k}$, the statement of the Theorem 1 is equivalent to the following Proposition 1 Let

$$
\hat{u}_{r}\left(t, y ; z_{1}, \ldots, z_{r}\right)=t^{1-r} U_{r}\left(t^{\frac{1}{3}}, t^{-\frac{2}{3}} y ; t^{-\frac{2}{3}} z_{1}, \ldots, t^{-\frac{2}{3}} z_{r}\right) .
$$

Then for all $r \geq 1$

$$
\hat{u}_{r}(t, y ; \mathbf{z})=\left(\frac{\partial}{\partial t}\right)^{r-1} \int_{0}^{1+\zeta(t)} \frac{y}{(1-4 s y)^{3 / 2}} \prod_{j=1}^{r}\left(\frac{1}{\sqrt{1-4 s z_{j}}}-1\right) d s .
$$


Proof. First, applying the transformation (28) to $U_{0}, U_{1}$ we find

$$
\begin{gathered}
\hat{u}_{0}(t, y)=\frac{1}{2}\left(t-\frac{y}{\sqrt{1+\zeta}}\right) \sqrt{1-4(1+\zeta) y}-\frac{t-y}{2} \\
\hat{u}_{1}(t, y, z)=\frac{y \sqrt{1-4(1+\zeta) z}}{2(y-z) \sqrt{1-4(1+\zeta) y}}-\frac{1}{2 \sqrt{1-4(1+\zeta) y}}-\frac{z}{2(y-z)} .
\end{gathered}
$$

It can be verified by explicit integration that $\hat{u}_{1}$ satisfies $(29)$.

Next, for all $r \geq 2$ (29) is equivalent to

$$
\hat{u}_{k}(t, y ; \mathbf{z})=\left(\frac{\partial}{\partial t}\right)^{r-2}\left\{\frac{y \zeta^{\prime}(t)}{(1-4(1+\zeta) y)^{3 / 2}} \prod_{j=1}^{r}\left(\frac{1}{\sqrt{1-4(1+\zeta) z_{j}}}-1\right)\right\} .
$$

From (18) we have

$$
\zeta^{\prime}=\frac{16(1+\zeta)^{5 / 2}}{2-\zeta}
$$

So

$$
\begin{aligned}
\hat{u}_{2}\left(t, y ; z_{1}, z_{2}\right)= & \frac{8 t(1+\zeta)^{5 / 2}}{1-\zeta / 2} \frac{y}{(1-4(1+\zeta) y)^{3 / 2}} \\
& \times\left(\frac{1}{\sqrt{1-4(1+\zeta) z_{1}}}-1\right)\left(\frac{1}{\sqrt{1-4(1+\zeta) z_{2}}}-1\right)
\end{aligned}
$$

satisfies (29) as well.

Now suppose that (29) holds for $r=0,1, \ldots, k-1$ for some $k \geq 3$, and let us show that it holds as well for $r=k$.

The equation (3) leads to the following equation on $\hat{u}_{k}$ :

$$
\begin{aligned}
\hat{u}_{k}(t, y ; \mathbf{z})= & \frac{t}{y}\left(\hat{u}_{k}(t, y ; \mathbf{z})-y \hat{l}_{k}(x ; \mathbf{z})\right) \\
& +\frac{1}{y} \sum_{\omega \subset I_{k}} \hat{u}_{|\omega|}\left(t, y ; \mathbf{z}_{\omega}\right) \hat{u}_{k-|\omega|}\left(t, y ; \mathbf{z}_{I_{k} \backslash \omega}\right) \\
& +\sum_{j=1}^{k}\left[\frac{1}{y-z_{j}}\left(\frac{z_{j}}{y} \hat{u}_{k-1}\left(t, y ; \hat{\mathbf{z}}_{j}\right)-\frac{y}{z_{j}} \hat{u}_{k-1}\left(t, z_{j} ; \hat{\mathbf{z}}_{j}\right)\right)\right. \\
& \left.+\hat{l}_{k-1}\left(t ; \hat{\mathbf{z}}_{j}\right)\right],
\end{aligned}
$$

with

$$
\hat{l}_{k}(t ; \mathbf{z})=[y] \hat{u}_{k}(t, y ; \mathbf{z})
$$


Rewrite (31) as

$$
\begin{aligned}
\left(y-t-2 \hat{u}_{0}(t, y)\right) \hat{u}_{k}(t, y, \mathbf{z})= & \sum_{\substack{\omega \subset I_{k} \\
1<|\omega|<k}} \hat{u}_{|\omega|}\left(t, y ; \mathbf{z}_{\omega}\right) \hat{u}_{k-|\omega|}\left(t, y ; \mathbf{z}_{I_{k} \backslash \omega}\right) \\
& +\sum_{j=1}^{k} \frac{y}{y-z_{j}}\left(\frac{z_{j}}{y} \hat{u}_{k-1}\left(t, y ; \hat{\mathbf{z}}_{j}\right)-\frac{y}{z_{j}} \hat{u}_{k-1}\left(t, z_{j} ; \hat{\mathbf{z}}_{j}\right)\right) \\
& +y\left(\sum_{j=1}^{k} \hat{l}_{k-1}\left(t ; \hat{\mathbf{z}}_{j}\right)-t \hat{l}_{k}(t ; \mathbf{z})\right)
\end{aligned}
$$

In order to apply the combinatorial identity (24) to sum over $\omega$ in (32), we need to introduce some new notation. We put

$$
\begin{aligned}
\lambda & =\frac{y \cdot \zeta^{\prime}}{(1-4(1+\zeta) y)^{3 / 2}}=\frac{16 y(1+\zeta)^{5 / 2}}{(2-\zeta)(1-4(1+\zeta) y)^{3 / 2}}, \\
f_{j} & =\frac{1}{\sqrt{1-4(1+\zeta) z_{j}}}-1,
\end{aligned}
$$

let $\mathbf{D}=\frac{\partial}{\partial t}$ and fix the following integrals

$$
\begin{aligned}
\mathbf{D}^{-1} \lambda & =\frac{1}{2 \sqrt{1-4(1+\zeta) y}}, \\
\mathbf{D}^{-2} \lambda & =\frac{(\zeta-8(1+\zeta) y) \sqrt{1-4(1+\zeta) y}}{16(1+\zeta)^{3 / 2}} \\
\mathbf{D}^{-1}\left(\lambda f_{j}\right) & =\frac{y \sqrt{1-4(1+\zeta) z_{j}}}{2\left(y-z_{j}\right) \sqrt{1-4(1+\zeta) y}}-\frac{1}{2 \sqrt{1-4(1+\zeta) y}}-\frac{z_{j}}{2\left(y-z_{j}\right)} .
\end{aligned}
$$

With these conventions we have

$$
\hat{u}_{0}(t, y)=\mathbf{D}^{-2}(\lambda)-\frac{(t-y)}{2}, \quad \hat{u}_{1}\left(t, y, z_{j}\right)=\mathbf{D}^{-1}\left(\lambda f_{j}\right),
$$

and we have supposed that, according to (30),

$$
\hat{u}_{r}\left(t, y ; \mathbf{z}_{\omega}\right)=\mathbf{D}^{r-2}\left(\lambda f_{\omega}\right)
$$

for all $\omega \subset I_{k}$ such that $r=|\omega|, 2 \leq r \leq k-1$. 
Now applying (24) we obtain

$$
\begin{aligned}
\sum_{\substack{\omega \subset I_{k} \\
1<|\omega|<k}} \hat{u}_{|\omega|}\left(t, y ; \mathbf{z}_{\omega}\right) \hat{u}_{k-|\omega|}\left(t, y ; \mathbf{z}_{I_{k} \backslash \omega}\right)+2 \mathbf{D}^{-2}(\lambda) \mathbf{D}^{k-2}\left(\lambda f_{I_{k}}\right) \\
=\sum_{\omega \subset I_{k}} \mathbf{D}^{|\omega|-2}\left(\lambda f_{\omega}\right) \mathbf{D}^{|\bar{\omega}|-2}\left(\lambda f_{\bar{\omega}}\right) \\
=2 \mathbf{D}^{k-2}\left\{\mathbf{D}^{-2}(\lambda) \cdot \lambda f_{I_{k}}\right\}-2(k-2) \mathbf{D}^{k-3}\left\{\mathbf{D}^{-1}(\lambda) \cdot \lambda f_{I_{k}}\right\} \\
\quad+2 \sum_{j=1}^{k} \mathbf{D}^{k-3}\left\{\mathbf{D}^{-1}\left(\lambda f_{j}\right) \cdot \lambda f_{I_{k} \backslash j}\right\} \\
=\quad 2 \mathbf{D}^{k-3}\left\{\mathbf{D}^{-2}(\lambda) \cdot \mathbf{D}(\lambda) f_{I_{k}}+3 \mathbf{D}^{-1}(\lambda) \cdot \lambda f_{I_{k}}\right. \\
\left.\quad+\sum_{j=1}^{k}\left(\mathbf{D}^{-2}(\lambda) \mathbf{D}\left(f_{j}\right)-\mathbf{D}^{-1}(\lambda) f_{j}+\mathbf{D}^{-1}\left(\lambda f_{j}\right)\right) \cdot \lambda f_{I_{k} \backslash j}\right\}
\end{aligned}
$$

where in the last equality we used the identities

$$
\mathbf{D}\left\{\mathbf{D}^{-2}(\lambda) \lambda f_{I_{k}}\right\}=\mathbf{D}^{-1}(\lambda) \cdot \lambda f_{I_{k}}+\mathbf{D}^{-2}(\lambda) \mathbf{D}(\lambda) f_{I_{k}}+\sum_{j=1}^{k} \mathbf{D}^{-2}(\lambda) \mathbf{D}\left(f_{j}\right) \cdot \lambda f_{I_{k} \backslash j}
$$

and

$$
k \mathbf{D}^{-1}(\lambda) \cdot \lambda f_{I_{k}}=\sum_{j=1}^{k} \mathbf{D}^{-1}(\lambda) f_{j} \cdot \lambda f_{I_{k} \backslash j}
$$

On the other hand, we have

$$
\begin{aligned}
& \frac{y}{y-z_{j}}\left(\frac{z_{j}}{y} \hat{u}_{k-1}\left(t, y, \hat{\mathbf{z}}_{j}\right)-\frac{y}{z_{j}} \hat{u}_{k-1}\left(t, z_{j}, \hat{\mathbf{z}}_{j}\right)\right) \\
& \quad=\mathbf{D}^{k-3}\left\{\left(\frac{z_{j}}{y-z_{j}}-\frac{y}{y-z_{j}} \frac{(1-4(1+\zeta) y)^{3 / 2}}{\left(1-4(1+\zeta) z_{j}\right)^{3 / 2}}\right) \cdot \lambda f_{I_{k} \backslash j}\right\}
\end{aligned}
$$

and

$$
y-t-2 \hat{u}_{0}(t, y)=-2 \mathbf{D}^{-2}(\lambda),
$$

so we further rewrite (32) as

$$
\begin{aligned}
-2 \mathbf{D}^{-2}(\lambda) \cdot & \hat{u}_{k}(t, y ; \mathbf{z})+2 \mathbf{D}^{-2}(\lambda) \cdot \mathbf{D}^{k-2}\left(\lambda f_{I_{k}}\right) \\
= & \mathbf{D}^{k-3}\left\{2\left(\mathbf{D}^{-2}(\lambda) \cdot \mathbf{D}(\lambda) f_{I_{k}}+3 \mathbf{D}^{-1}(\lambda) \cdot \lambda f_{I_{k}}\right)\right. \\
& +\sum_{j=1}^{k} \lambda f_{I_{k} \backslash j} \cdot\left(2 \mathbf{D}^{-2}(\lambda) \cdot \mathbf{D}\left(f_{j}\right)-2 \mathbf{D}^{-1}(\lambda) f_{j}+2 \mathbf{D}^{-1}\left(\lambda f_{j}\right)\right. \\
& \left.\left.+\frac{z_{j}}{y-z_{j}}-\frac{y}{y-z_{j}} \frac{(1-4(1+\zeta) y)^{3 / 2}}{\left(1-4(1+\zeta) z_{j}\right)^{3 / 2}}\right)\right\} \\
& +y\left(\sum_{j=1}^{k} \hat{l}_{k-1}\left(t ; \hat{\mathbf{z}}_{j}\right)-t \hat{l}_{k}(t ; \mathbf{z})\right) .
\end{aligned}
$$


A straightforward calculation then shows that

$$
\begin{gathered}
\mathbf{D}^{-2}(\lambda) \cdot \mathbf{D}(\lambda)+3\left(\mathbf{D}^{-1} \lambda\right) \cdot \lambda=\frac{96(1+\zeta)^{5 / 2}}{(2-\zeta)^{2}} y \\
2 \mathbf{D}^{-2}(\lambda) \cdot \mathbf{D}\left(f_{j}\right)-2 \mathbf{D}^{-1}(\lambda) \cdot f_{j}+2 \mathbf{D}\left(-1\left(\lambda f_{j}\right)\right. \\
+\left(\frac{z_{j}}{y-z_{j}}-\frac{y}{y-z_{j}} \frac{(1-4(1+\zeta) y)^{3 / 2}}{(1-4(1+\zeta) z)^{3 / 2}}\right) \\
=\frac{16(1+\zeta)^{5 / 2}(\zeta-2+8(1+\zeta) z)}{(2-\zeta)^{2}(1-4(1+\zeta) z)^{3 / 2}} y
\end{gathered}
$$

It follows from (35), (36) that the right-hand side of (34) is a linear function of $y$. On the other hand, the left-hand side of (34) turns to zero both at $y=0$, because $\hat{u}_{k}(t, 0 ; \mathbf{z})=0$ and $\left.\mathbf{D}^{2-k} \lambda f_{I_{k}}\right|_{y=0}=0$, and at $y=\zeta /(8(1+\zeta))$, because then $\mathbf{D}^{-2}(\lambda)=0$.

Thus both sides of (34) are identically zero, which implies $\hat{u}_{k}=\mathbf{D}^{k-2}\left(\lambda f_{I_{k}}\right)$. This finishes the proof of both the proposition and Theorem 1 .

\section{References}

[1] D. Arqués and A. Giorgetti. Counting rooted maps on a surface. Theoretical Computer Science, 234:255-272, 2000.

[2] E.A. Bender and E.R. Canfield. The asymptotic number of rooted maps on a surface. Journal of Combinatorial Theory Series A, 43(2):244-257, 1986.

[3] Zhi-Cheng Gao. The numbers of rooted triangular maps on a surface. J. Comb. Theory Ser. B, 52(2):236-249, 1991.

[4] Zhi-Cheng Gao. The asymptotic number of rooted 2-connected triangular maps on a surface. J. Comb. Theory Ser. B, 54(1):102-112, 1992.

[5] B.J. Durhuus J. Ambjørn and T. Jonsson. Quantum geometry: A Statistical Field Theory Approach. Cambridge Univ. Press, 1997.

[6] M. Krikun. Boundaries of random triangulation of a disk. Discrete Mathematics and Applications, 14(3):301-315, 2003.

[7] E. Nemeth R.C. Mullin and P.J. Schellenberg. The enumeration of almost cubic maps. Proceedings of the Louisiana Conference on Combinatorics, Graph Theory and Computer Science, 1:281-295, 1970.

[8] N.J.A. Sloane. The On-Line Encyclopedia of Integer Sequences. http://www.research.att.com/ njas/sequences/.

[9] W.T. Tutte. A census of planar triangulations. Canad. J. Math, 14(1):21-38, 1962.

[10] W.T. Tutte. A census of slicings. Canad. J. Math, 14(4):708-722, 1962. 